\title{
Optimal Trajectory Planning and Linear Velocity Feedback Control of a Flexible Piezoelectric Manipulator for Vibration Suppression
}

\author{
Junqiang Lou, ${ }^{1}$ Yanding Wei, ${ }^{2}$ Guoping Li, ${ }^{1}$ Yiling Yang, ${ }^{2}$ and Fengran Xie ${ }^{2}$ \\ ${ }^{1}$ Zhejiang Provincial Key Lab of Part Rolling Technology, College of Mechanical Engineering and Mechanics, Ningbo University, \\ Ningbo 315211, China \\ ${ }^{2}$ China Key Laboratory of Advanced Manufacturing Technology of Zhejiang Province, School of Mechanical Engineering, \\ Zhejiang University, Hangzhou 310027, China
}

Correspondence should be addressed to Junqiang Lou; loujunqiang@nbu.edu.cn

Received 26 June 2015; Revised 4 August 2015; Accepted 5 August 2015

Academic Editor: Mario Terzo

Copyright (C) 2015 Junqiang Lou et al. This is an open access article distributed under the Creative Commons Attribution License, which permits unrestricted use, distribution, and reproduction in any medium, provided the original work is properly cited.

\begin{abstract}
Trajectory planning is an effective feed-forward control technology for vibration suppression of flexible manipulators. However, the inherent drawback makes this strategy inefficient when dealing with modeling errors and disturbances. An optimal trajectory planning approach is proposed and applied to a flexible piezoelectric manipulator system in this paper, which is a combination of feed-forward trajectory planning method and feedback control of piezoelectric actuators. Specifically, the joint controller is responsible for the trajectory tracking and gross vibration suppression of the link during motion, while the active controller of actuators is expected to deal with the link vibrations after joint motion. In the procedure of trajectory planning, the joint angle of the link is expressed as a quintic polynomial function. And the sum of the link vibration energy is chosen as the objective function. Then, genetic algorithm is used to determine the optimal trajectory. The effectiveness of the proposed method is validated by simulation and experiments. Both the settling time and peak value of the link vibrations along the optimal trajectory reduce significantly, with the active control of the piezoelectric actuators.
\end{abstract}

\section{Introduction}

Flexible manipulators have been used in various applications including field inspection, space exploration, medical application, and hazardous environment. Compared with their rigid counterparts, flexible manipulators exhibit many advantages such as higher manipulation speed, greater payload to weight ratio, and lower energy consumption. Moreover, they can be quickly adapted to changing situations and product design variations [1]. However, the conflicting requirements between high speed and high positioning accuracy have made the control of flexible manipulators a real challenging problem. Structural flexibility leads to the appearance of undesirable vibrations during motion. So flexible manipulator system exhibits combined behaviors of rigid body motions and vibrations [2]. These oscillations lead to deterioration of positioning accuracy and efficiency, making the control of such system extremely difficult.

To address these problems, quite a lot of researches have been conducted on the vibration control of flexible manipulators. The existing vibration control techniques can be broadly classified into two categories, namely, feedback control and feed-forward control. The advantage of feed-forward control is that it does not require any additional sensors or actuators. Hence, the control system can be manufactured at low costs, thus being more economical. One special feed-forward control strategy, known as input command shaping, has been studied widely since its first appearance [3]. Mohamed et al. [4] presented investigations into vibration control of a flexible manipulator using input shaping techniques with 
positive and negative input shapers. Alam and Tokhi [5] developed the design of a command shaping controller for vibration suppression of a flexible manipulator, using multiobjective genetic optimization process. More recently, Cole and Wongratanaphisan [6] described an adaptive FIR input command shaping methodology to achieve zero residual vibration control of a rigid-flexible manipulator system. The input command shaping method is effective to ensure that the input does not excite vibrations of the manipulator. But it usually costs a large amount of computation when dealing with multiple vibration modes. And it cannot suppress the induced and existing vibrations.

An alternative feed-forward control strategy to achieve vibration control of flexible manipulators is the trajectory planning approach. Wu et al. [7] proposed an optimal trajectory planning method for vibration reduction of a dual arm space robot with front flexible links, in which the trajectory was described using a fourth-order quasiuniform B-spline. Heidari et al. [8] considered the problem on the rest-to-rest motion of a flexible manipulator and formulated the trajectory planning problem as a Pontryagin optimal problem. Springer et al. [9] focused on timeoptimal trajectory planning for robots with flexible links and determined the minimum time trajectory for avoiding elastic vibration of the link. Choi et al. [10] described a new trajectory planning method for output tracking of linear flexible system using exact equilibrium manifolds. However, the trajectory planning technique is a feed-forward control strategy; vibration modes of the flexible manipulator are still controlled in open loop [11]. When dealing with various disturbances and parameters variations, there would still be large residual vibrations that need to be controlled [12].

To overcome the drawbacks of feed-forward control schemes, many researchers have tried to solve the problems of link vibration suppression using feedback control techniques. Among them, some researchers concentrated on reducing the link vibrations using joint motor [13]. However, the existence of nonminimum phase dynamics between the tip position and the input torque applied at the joint motor makes the system difficult to stabilize [14]. A rather different approach for feedback control of link vibrations has been developed in recent years, relying on the use of smart materials [15]. Among those smart materials, piezoelectric (PZT) materials have been found extensive applications in structure vibration control, due to their lightweight, fast response, large bandwidth, and so on [16]. Sun et al. [17] realized the rigid motion control and vibration damping of a flexible manipulator, using a combined scheme of a PD controller for the joint motor and a linear velocity feedback (L-type) controller for the piezoelectric actuators. Gurses et al. [18] extended the previous work studied and introduced a serial array of fiber optic sensors to the control of flexible manipulators which allowed simultaneous linear (L-type) and angular (A-type) velocity feedback. Li et al. [19] presented a study on vibration suppression of a flexible piezoelectric manipulator using adaptive fuzzy sliding mode control. Choi et al. [20] studied the position control of a single-link flexible manipulator and designed a hysteresis compensator for the piezoelectric actuators.
However, in most studies on active control of flexible manipulator, attention seems to be focused on how to improve the performance of the piezoelectric controller. The contributions of the feed-forward torque or the required trajectory planning are rarely considered. A more reasonable and efficient approach seems to emerge if a combined strategy is considered. Specifically, the joint motor is responsible for the gross vibrationless motion of the structure through trajectory planning approach, while the PZT actuators are used to deal with vibrations that may arise from modeling errors and external disturbances after joint motion, by means of active control. The remainder of this paper is organized as follows. In Section 2, the flexible piezoelectric manipulator system is introduced and the governing equations of the system are derived. Section 3 proposes the optimal trajectory planning approach. The joint angle of the link is expressed as a quintic polynomial function. The constraint conditions, the initial and final conditions of the joint angle, angular velocity, and angular acceleration are applied. The sum of the link vibration energy is chosen as the objective function. Then, the optimal trajectory planning algorithm is constructed by the use of genetic algorithm. In Section 4, numerical analysis and simulations are conducted. The optimal trajectory of the flexible piezoelectric manipulator in a point-to-point motion is obtained. Section 5 describes an experimental apparatus of the flexible manipulator system, together with the experimental results, before concluding in Section 6 .

\section{Dynamic Modeling}

The schematic diagram of the flexible piezoelectric manipulator system is shown in Figure 1. It is composed of a rotary joint, a single-link flexible manipulator with a rectangular cross section and a pair of collocated piezoelectric (PZT) actuators. The manipulator is attached to the hub in such a way that it rotates in the horizontal plane only. The effect of gravity can be ignored, so the link can be modeled as an EulerBernoulli beam. The axis ox is the inertial reference line. The axis $o x$ is the tangential line to the neutral axis of the beam at the hub. For the sake of simplicity, the same assumptions are made as [17].

It is assumed that the dynamic behavior of the flexible manipulator during a point-to-point motion is dominated by the first several vibration modes, so the contributions of the higher vibration modes are negligible. Using assumed mode method, the deflection $w(x, t)$ of the link can be represented as

$$
w(x, t)=\sum_{i=1}^{m} \varphi_{i}(x) q_{i}(t)=\boldsymbol{\Phi}(x) \mathbf{q}(t)
$$

where $\varphi_{i}(x)$ and $q_{i}(t)$ devote the $i$ th mode shape function and corresponding generalized coordinate, respectively. $\Phi(x)=$ $\left[\varphi_{1} \cdots \varphi_{i} \cdots \varphi_{m}\right]$ and $\mathbf{q}(t)=\left[q_{1} \cdots q_{i} \cdots q_{m}\right]$ represent the mode shape function and generalized coordinate index, respectively. $m$ is the mode number. 


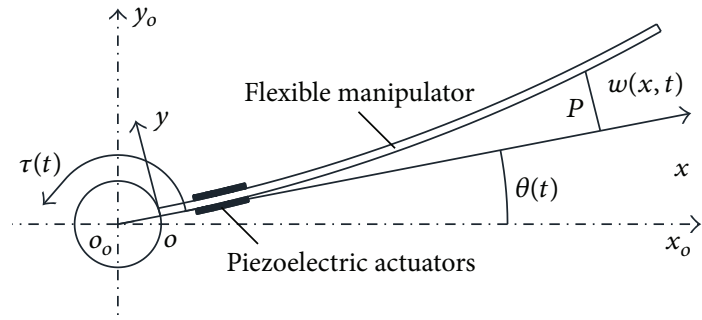

FIGURE 1: Schematic diagram of the flexible manipulator system.

Extended Hamilton's principle is used to derive the system equation. The derivation process and parameter expressions are explicitly presented in Appendix. The governing equations of the system can be derived as

$$
\begin{aligned}
& I_{\theta \theta} \ddot{\theta}+\mathbf{q}^{T} \mathbf{m}_{q q} \mathbf{q} \ddot{\theta}+2 \dot{\mathbf{q}}^{T} \mathbf{m}_{q q} \mathbf{q} \dot{\theta}+\mathbf{m}_{\theta q} \ddot{\mathbf{q}}=\tau(t), \\
& \mathbf{m}_{q q} \ddot{q}+\mathbf{m}_{\theta q}^{T} \ddot{\theta}+\mathbf{K}_{q} \mathbf{q}-\mathbf{m}_{q q} \mathbf{q} \dot{\theta}^{2}=c V(t)\left[\widehat{\boldsymbol{\Phi}}^{\prime}\right]^{T}\left(x_{s}\right) .
\end{aligned}
$$

It should be noticed that (2) represents the rigid motion of the piezoelectric manipulator, while (3) describes the dynamic behavior of the flexible link with the control of PZT actuators. If the desired trajectory can be realized by means of tracking control, for example, trajectory control can be realized when the servo motor works in the speed control mode. In the case that tracking control is possible, only (3) is used for planning a trajectory where the elastic vibration of the link is eliminated or minimum. Augmenting the proportional damping, the dynamic equation of the flexible manipulator system can be expressed as

$$
\begin{gathered}
\mathbf{m}_{q q} \ddot{\mathbf{q}}+\mathbf{m}_{\theta q}^{T} \ddot{\theta}+\mathbf{C}_{q} \dot{\mathbf{q}}+\mathbf{K}_{q} \mathbf{q}-\mathbf{m}_{q q} \mathbf{q} \dot{\theta}^{2} \\
=c V(t)\left[\widehat{\boldsymbol{\Phi}}^{\prime}\right]^{T}\left(x_{s}\right) .
\end{gathered}
$$

It is known that it is feasible to use linear velocity feedback in the controller formulation. Thus, the linear velocity feedback (L-type) approach is readily implementable. Furthermore, the stability of the close-loop system including the L-type controller has been confirmed by Sun et al. in [17], as well as the effectiveness of the controller. Therefore, the linear velocity feedback (L-type) approach is employed to design the applied voltage of the PZT actuators in this study. In the L-type control strategy, the control voltage of the actuators is defined as

$$
V(t)=-k_{L}\left[\dot{w}\left(x_{s}+l_{p}\right)-\dot{w}\left(x_{s}\right)\right]=-k_{L} \widehat{\Phi}\left(x_{s}\right) \dot{\mathbf{q}},
$$

where $k_{L}$ is a positive control gain. Then, substituting (5) into (4) leads to

$$
\begin{aligned}
\mathbf{m}_{q q} \ddot{\mathbf{q}} & +\mathbf{m}_{\theta q}^{T} \ddot{\theta}+\mathbf{C}_{q} \dot{\mathbf{q}}+c k_{L}\left[\widehat{\boldsymbol{\Phi}}^{\prime}\right]^{T}\left(x_{s}\right) \widehat{\boldsymbol{\Phi}}\left(x_{s}\right) \dot{\mathbf{q}}+\mathbf{K}_{q} \mathbf{q} \\
& -\mathbf{m}_{q q} \mathbf{q} \dot{\theta}^{2}=0 .
\end{aligned}
$$

It can be observed from (6) that the rigid motion of the joint, elastic vibration of the link, and active control of the PZT actuators are all considered in the procedure of trajectory planning. However, it is known that the piezoelectric parameters are very small numbers, which means that the contribution assured by the PZT control is relatively less than that of the feed-forward trajectory planning during joint motion. Therefore, the essence of the optimal trajectory planning approach is that the feed-forward trajectory planning is responsible for the gross vibrationless motion of the structure during joint motion, while the PZT actuators are used to deal with vibrations after joint motion, by means of L-type control strategy.

\section{Optimal Trajectory Planning}

This study focuses on the problem related to a point-to-point (PTP) motion of the flexible manipulator system rotating from the initial joint angle $\theta_{0}$ to the final joint angle $\theta_{f}$ over a fixed traveling time $t_{f}$ [21]. In practice, elastic vibrations of the flexible link are unavoidable due to the flexible-rigid coupled dynamics. Considering the presence of parameter uncertainties and external disturbances, the residual vibrations of the link are still exciting at the end of PTP motion, in spite of active control of PZT actuators. For a given PTP maneuver in joint space, the objective is to find an optimal joint trajectory that minimizes link vibrations during and after joint motion. The total vibration energy of a trajectory is adopted as the performance index, which is defined as

$$
\mathrm{PI}(x)=\lambda_{1} \int_{t_{0}}^{t_{f}} \mathbf{q}^{T}(t) \mathbf{q}(t) d t+\lambda_{2} \int_{t_{f}}^{t_{d}} \mathbf{q}^{T}(t) \mathbf{q}(t) d t,
$$

where $t_{d}$ is the settling time of the link vibrations with piezoelectric control. The first part in (7) represents vibration energy of the link during joint motion, while the second part represents residual vibration energy after joint motion. $\lambda_{1}$ and $\lambda_{2}$ are the weighting indexes of the two parts, respectively.

To realize a smooth motion, smooth and continuous functions are typically adopted to plan the joint trajectory. Moreover, the first and second derivatives of the trajectory are also expected to be smooth and continuous. Among those common trajectory functions, the quintic polynomial function has the advantages of being accurate, precise, and easy to design and control. Moreover, it has the extra advantage of controlling the acceleration at the initial point and goal [22]. So the quintic polynomial function is selected to interpolate the optimal trajectory curves, which can be written as

$$
\theta(t)=C_{0}+C_{1} t+C_{2} t^{2}+C_{3} t^{3}+C_{4} t^{4}+C_{5} t^{5},
$$

where $C_{0}, C_{1}, C_{2}, C_{3}, C_{4}$, and $C_{5}$ are the coefficients to be determined from the initial and final boundary conditions. To get a smooth motion, the initial angle, angular velocity, and angular acceleration are set to be zero. Meanwhile, the final angular velocity and angular acceleration are also set to be zero.

To get the optimal trajectory that minimizes the performance index in (7), the travelling time $t_{f}$ is divided into $n$ divisions, each of which has an equal time interval. 


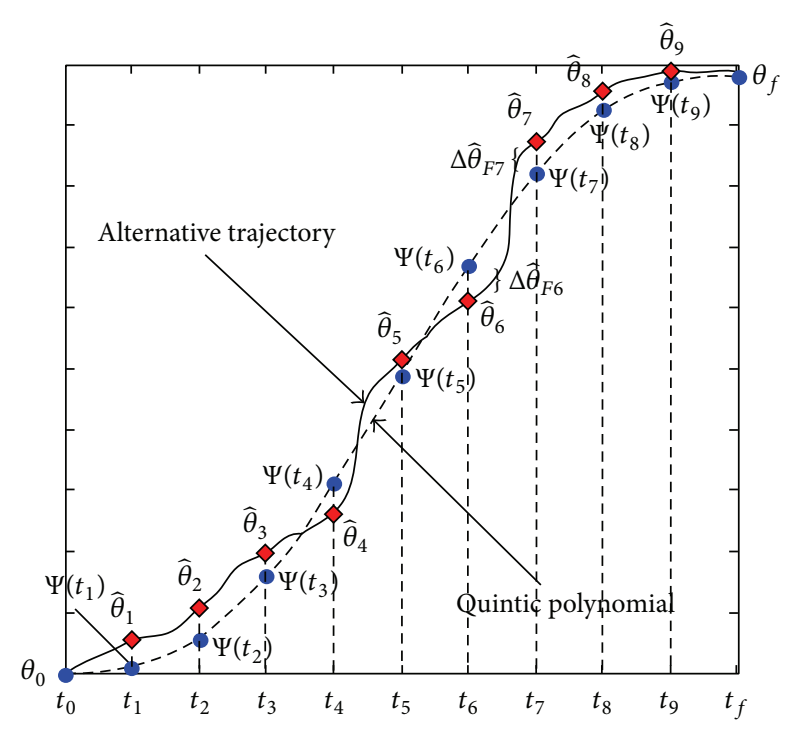

FIgURE 2: Discrete joint angle of the flexible manipulator system $\widehat{\theta}_{i}$ $(n=10)$.

If the times at divided knots can be denoted by $t_{0}(=0)$, $t_{1}, \ldots, t_{i}, \ldots, t_{n-1}, t_{f}$, the joint angle at $t_{i}$ is denoted by $\widehat{\theta}_{i}$. To construct the optimal trajectory, the quintic polynomial function is employed to interpolate the discrete angles. The angular velocity and angular acceleration are obtained by differentiating the quintic polynomial curves with respect to time. Finally, the discrete angels of the link are defined by introducing parameters $\Delta \widehat{\theta}_{F i}$ as

$$
\widehat{\theta}_{i}=\Psi\left(t_{i}\right)+\Delta \widehat{\theta}_{F i},
$$

where $\Psi(t)$ represents the quintic polynomial motion with the above boundary conditions.

Consequently, the parameter $\Delta \widehat{\theta}_{F i}$ denotes the deviation from the trajectory for the quintic polynomial motion. The discrete joint angles of the flexible link are shown in Figure 2.

Genetic algorithm (GA) approach is gaining popularity for solving complex problems in robotics. It is a populationbased stochastic and global search method. Its performance is superior to that revealed by classical optimization techniques and has been successfully used in robot trajectory planning technique [23]. As the procedure discussed above, it is possible to express the optimal trajectory $\widehat{\theta}_{i}$ by using the parameter $\Delta \widehat{\theta}_{F i}$, whose number is $n-1$ for the $n$ divisions of $t_{f}$. The trajectory planning procedures of the link using GA are carried out as follows. The parameters $\Delta \widehat{\theta}_{F i}(i=1,2, \ldots, n-1)$ are considered to be optimized. First, the angular velocity $\dot{\hat{\theta}}(t)$ and angular acceleration $\ddot{\widetilde{\theta}}(t)$ are obtained from the quintic polynomial interpolation of the discrete joint angles $\widehat{\theta}_{i}$. Then, the dynamic response of the flexible piezoelectric manipulator is calculated through numerical integration of (6). Finally, the optimal trajectory can be derived through minimizing the performance index in (7), using genetic algorithm. The main steps are summarized as below.
Step 1. Division number $n$ is determined; the discrete angles of the quintic polynomial motion $\left[\psi\left(t_{1}\right), \psi\left(t_{2}\right), \ldots, \psi\left(t_{i}\right)\right.$, $\left.\ldots, \psi\left(t_{n-1}\right)\right]$ are calculated. Meanwhile, the parameters of the GA are also determined.

Step 2. The initial population for the first generation is generated through a random generator. Every individual includes $n-1$ genes corresponding to the interior points to be selected, which is defined as $\mathbf{x}_{k}=\left[x_{k 1}, x_{k 2}, \ldots, x_{k i}, \ldots, x_{k(n-1)}\right]$. The gene of the individual $x_{k i}$ represents the optimized parameter $\Delta \widehat{\theta}_{F i}$, and the dimension of the search space is $n-1$.

Step 3. The trajectory of the link is generated through the quintic polynomial interpolation, and the vibration response of the link is derived through numerical simulations. The fitness values $\mathrm{PI}(x)$ of all the individuals in (7) are evaluated.

Step 4. A set of genetic operators, which are selection, crossover, and mutation, are used in succession to create a new population of chromosomes for the next generation.

Step 5. The process of evaluation and creation of new successive generations is repeated until the termination condition is satisfied. And the optimal trajectory for vibration suppression is finally decided where the fitness value $\operatorname{PI}(x)$ is minimum.

\section{Simulation Studies}

Numerical simulations are carried out to evaluate the performance of the proposed trajectory planning approach using MATLAB. The parameters of the proposed flexible manipulator system are shown in Table 1. In simulation, the actuator pairs are chosen to lie near the root of the link, where the strain energy of the structure is the highest [24]. For simplicity, only the first vibration mode is taken into account, which is found to be at $\omega_{1}=24.5 \mathrm{rad} / \mathrm{s}(3.9 \mathrm{~Hz})$, with modal damping $\zeta_{1}=0.02$. And the parameters of the GA are given in Table 2. The weighting indexes of the vibration energy during and after joint motion are set to $\lambda_{1}=0.25, \lambda_{2}=0.75$.

A fast motion case is considered, here. The traveling time $t_{f}$ is set to be 0.5 seconds. The initial and final angles of the joint are set to be $0 \mathrm{rad}$ and $\pi / 2 \mathrm{rad}$, respectively. And the number of trajectory partitions $n$ is set to be 10. Meanwhile, to speed up the convergence of GA search, the search space of the optimized parameters $\Delta \widehat{\theta}_{F i}$ is taken to be

$$
\Delta \widehat{\theta}_{F i} \in\left[-0.5\left|\psi\left(t_{i}\right)-\theta_{f}\right| 0.5\left|\psi\left(t_{i}\right)-\theta_{f}\right|\right] .
$$

For a real flexible manipulator system, the joint acceleration, the torque of the motor, and control voltage of the PZT actuator should have constraint values [25]. The constraint conditions of the flexible manipulator system are defined as

$$
\begin{aligned}
0 & <\widehat{\theta}_{i}<\theta_{f} \quad(i=1,2, \ldots, n-1), \\
\ddot{\theta} & \leq \ddot{\theta}_{\max }, \\
V & \leq V_{\max }
\end{aligned}
$$

where the parameter $V_{\max }$ is set to be $150 \mathrm{~V}$ and the maximum of the joint acceleration $\ddot{\theta}_{\max }$ is assumed to be $80 \mathrm{rad} / \mathrm{s}^{2}$. 
TABle 1: Parameters of the flexible manipulator system.

\begin{tabular}{lcc}
\hline Parameter & Flexible link & PZT actuator \\
\hline Material & Epoxy & PZT-5 \\
Length $(\mathrm{mm})$ & 620.0 & 40.0 \\
Width $(\mathrm{mm})$ & 30.0 & 10.0 \\
Thickness $(\mathrm{mm})$ & 3.0 & 0.8 \\
Young's modulus $(\mathrm{Gpa})$ & 34.6 & 117.0 \\
Density $\left(\mathrm{Kg} / \mathrm{m}^{3}\right)$ & 1840.0 & 7500.0 \\
PZT coefficient $d_{31}(\mathrm{C} / \mathrm{N})$ & N/A & $187 \times 10^{-12}$ \\
\hline
\end{tabular}

TABLE 2: Parameters of genetic algorithm.

\begin{tabular}{lc}
\hline Parameter & Value \\
\hline Population type & Binary string \\
Number of genes & 48 bits \\
Population size & 25 \\
Crossover ratio & 0.6 \\
Mutation ratio & 0.05 \\
Generations & 100 \\
\hline
\end{tabular}

The evolution history of the GA search is shown in Figure 3. It can be seen that the fitness reduces with an increasing value of the generation number. And the optimal trajectory can be approximately obtained at the 40th generation. The results of numerical calculations and simulations are presented in Figure 4. The angular displacement, velocity, and acceleration of the obtained optimal trajectory are depicted in Figures 4(a)-4(c), respectively. The tip vibration response of the flexible link along the optimal trajectory is illustrated in Figure 4(d). For comparison, the simulation results along the quintic polynomial trajectory are also presented, correspondingly.

As seen in Figure 4(d), dynamic response of the link along the optimal trajectory shows nearly no trace of oscillations after joint motion. That is to say, there is nearly no residual vibration in the flexible link (i.e., the modal displacement after $0.5 \mathrm{~s}$ ), while the dynamic response of the link along the quintic polynomial trajectory owns large oscillations all through the simulation interval. The presence of residual vibrations after joint motion is clearly visible, even after a period of 3-fold traveling time $t_{f}$.

\section{Experimental Results}

A photograph of the experimental setup is shown in Figure 5. The flexible piezoelectric manipulator is driven by an AC servo motor (Yaskawa SGMAH01A1A2S) with a reduction gear (Harmonic Drive CSF-17-50-2A) 50:1. A built-in 16-bit incremental encoder is installed within the motor to measure the rotation angle. The link is clamped at the shaft of the reduction gear through a coupling and is limited to rotate only in the horizontal plane. Meanwhile, a pair of strain gauges assembled in half-bridge configuration (resistance $120 \Omega$, sensitivity 2.08) are placed at the base of the link to

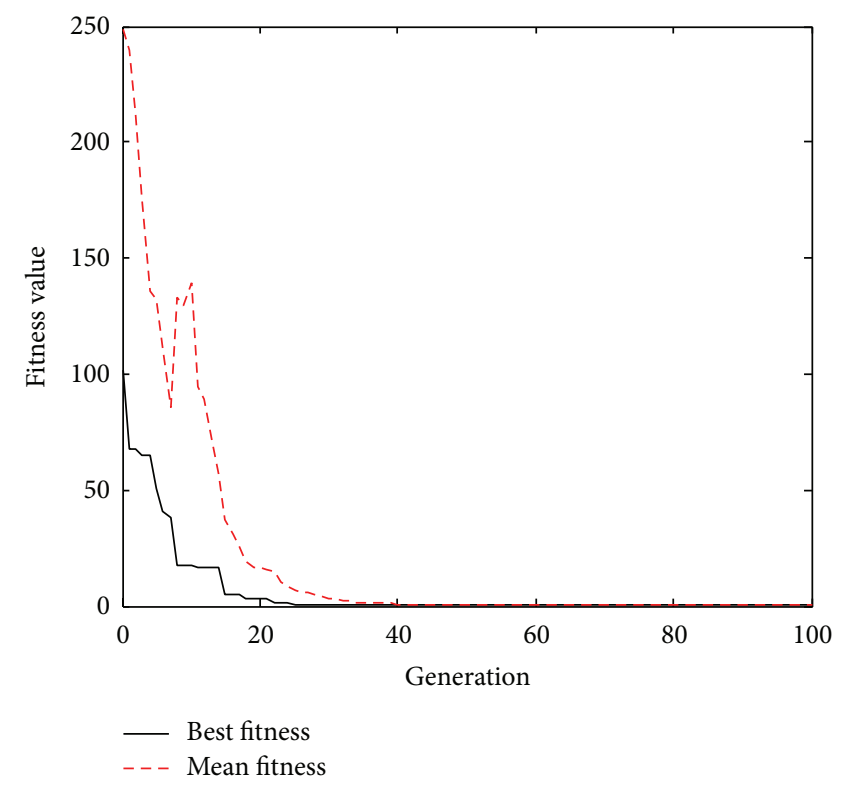

FIgURE 3: Evolution history of the genetic algorithm.

measure the vibrations. A pair of piezoelectric patches (PZT$5 \mathrm{~A}$ ) are attached to the link for the vibration suppression, located close to the strain sensor to get the best control effect.

The control system is realized with an industrial computer. A DAQ device (Advantech PCI-1742U) is used, providing multichannel $\mathrm{D} / \mathrm{A}, \mathrm{A} / \mathrm{D}$, and counter modules for data acquisition and control output. For joint motion control, the AC servomotor is operated in the speed control mode. The output signal of the encoder is fed back to the industrial computer through the counter module of the DAQ. And the input torque determined from the PD controller is applied to the motor through the D/A converter and servo driver (Yaskawa SGDH01AE). Meanwhile, the vibration signal of the flexible manipulator is low-pass filtered and amplified through a strain amplifier, which can amplify the signal to a voltage range of $-10 \mathrm{~V}$ to $10 \mathrm{~V}$, and then fed into the industrial computer through an A/D converter. To suppress the link vibrations actively, the input voltage determined from the controller, amplified 15 times with a power amplifier, is supplied to the PZT actuators through a D/A converter. The schematic diagram of the experimental setup is shown in Figure 6.

The first experiment was conducted to rotate the link from $0 \mathrm{rad}$ to $\pi / 2 \mathrm{rad}$ in 0.5 seconds along the quintic polynomial trajectory. But the active control of PZT actuators was not applied. Experimental results are presented in Figure 7. The angular displacement, velocity, and acceleration of the quintic polynomial trajectory are depicted in Figures 7(a)7 (c), respectively. The measured vibration response of the link by the strain gauges is shown in Figure 7(d).

As seen in Figures 7(a) and 7(b), the experimental results of joint angle and angular velocity are in perfect agreement with the reference curves. Thus, it can be inferred that the joint reference trajectory of the quintic polynomial was 


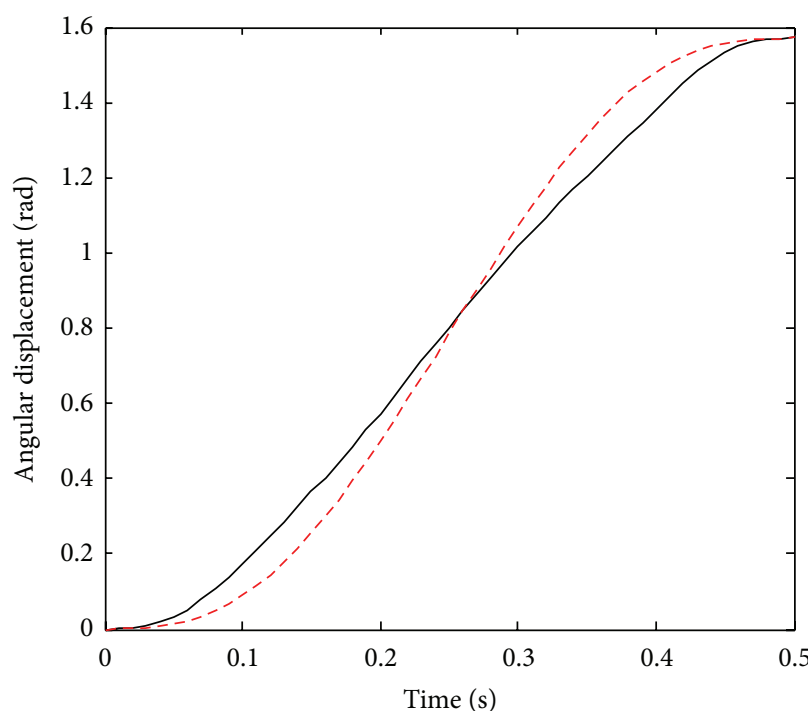

- Optimal trajectory

- - Quintic polynomial

(a) Angular displacement

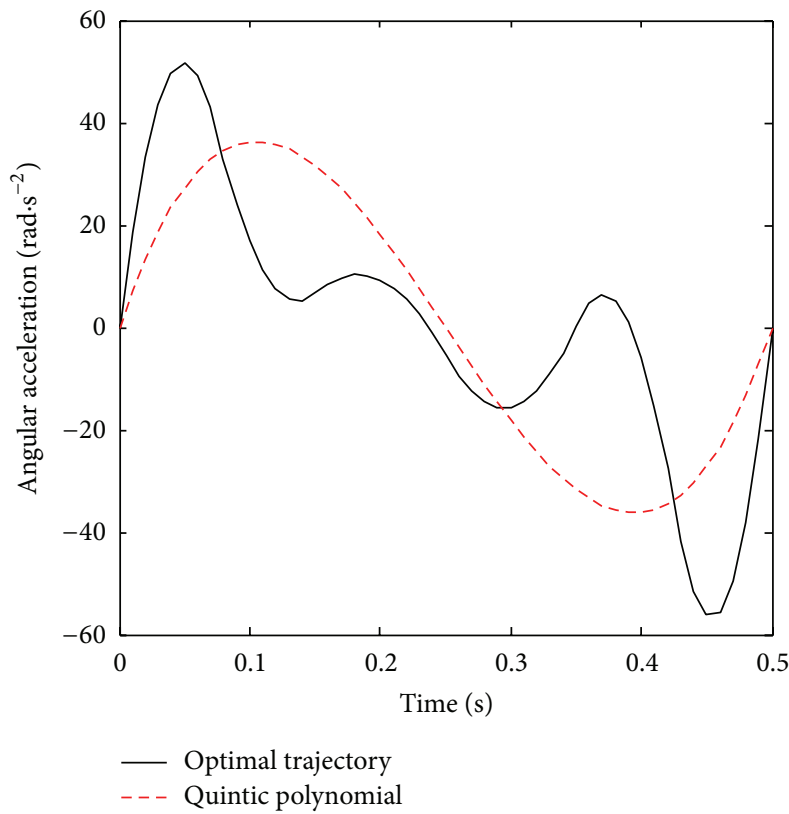

(c) Angular acceleration

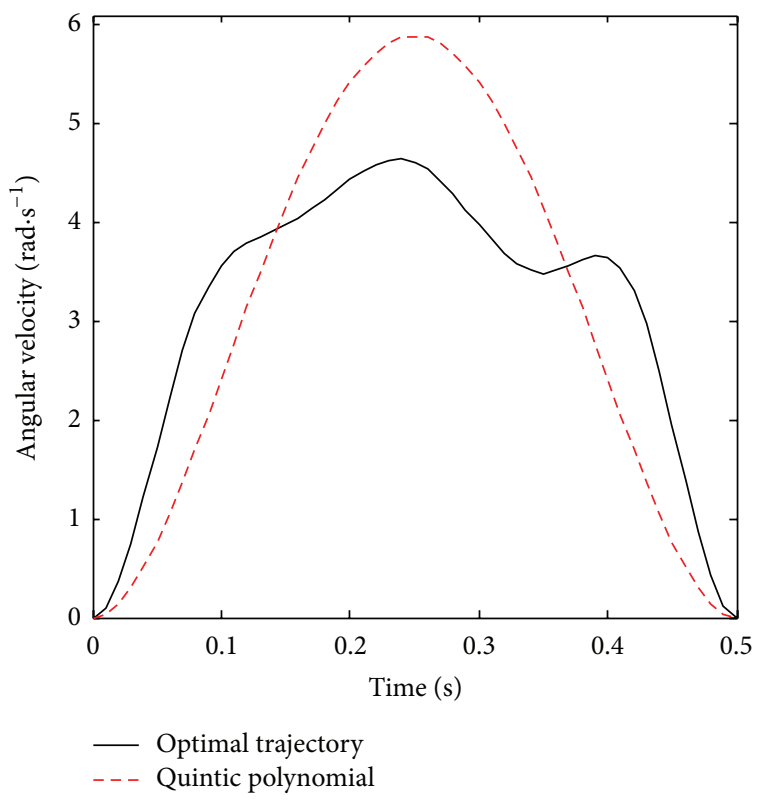

(b) Angular velocity

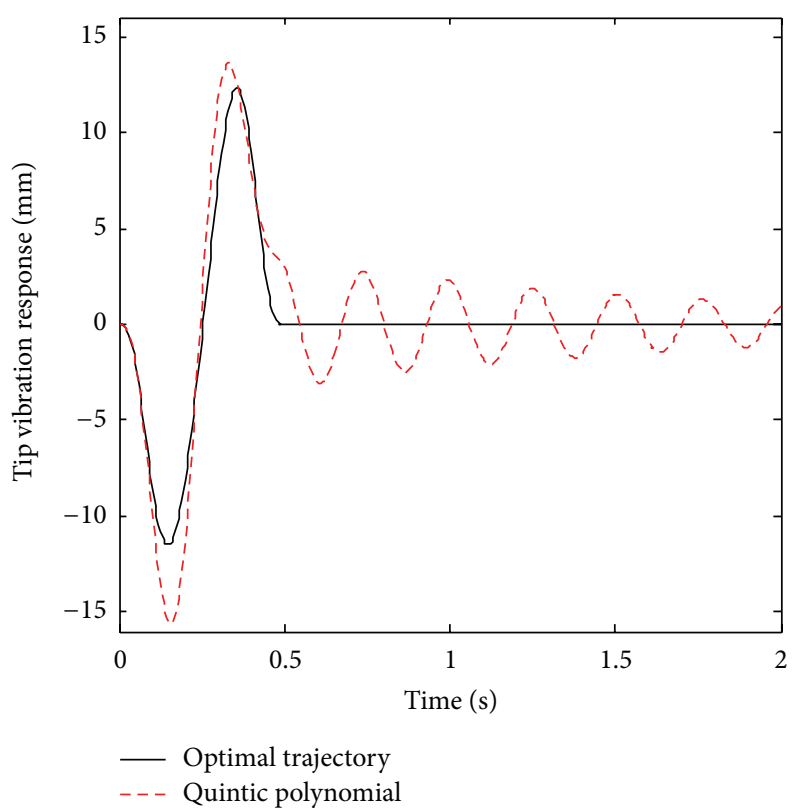

(d) Modal displacement

FIGURE 4: Comparison of simulation results between the optimal trajectory and quintic polynomial trajectory.

closely followed. However, unwanted oscillations with large amplitude both during and after the joint motion were clearly observed. As shown in Figure 7(d), the peak value of the measured vibration response exceeds the range of the $A / D$ converter, even after the joint motor has stopped for a period of $1 \mathrm{~s}$. Furthermore, the settling time of the uncontrolled link vibrations, which is defined as the damping period when the peak value of the sensor voltage does not exceed $0.1 \mathrm{~V}$, is found to be nearly 20 times longer than that of the motor motion $(0.5 \mathrm{~s})$, reaching $9.5 \mathrm{~s}$. Thus, vibration suppression of the link is necessary to attain a high positioning accuracy and efficiency. 


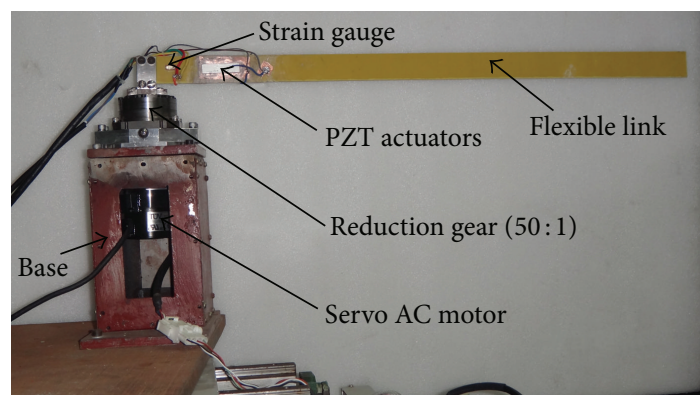

FIGURE 5: Photograph of the experimental setup.

The following experiment was the implementation of the optimal trajectory approach. But vibration control of the PZT actuators was still not applied. Figures 8(a)-8(d) show the angular displacement, velocity, acceleration, and measured vibration response of the link along the optimal trajectory, respectively. The link vibrations obtained from the quintic polynomial trajectory are compared with those obtained from the optimal trajectory strategy, as depicted in Figure 8(d). It can be observed from Figures 8(a) and 8(b) that experimental results of the angular displacement and velocity fairly coincide with the desired curves. Therefore, it can be confirmed that the trajectory tracking control of the flexible manipulator system is realized. Although the peak value of the link vibrations still exceeds the range of the $\mathrm{A} / \mathrm{D}$ converter during the joint motion, residual vibrations corresponding to the optimal trajectory are significantly less than those caused by the quintic polynomial trajectory. The settling time of the link vibrations reduces to $4.3 \mathrm{~s}$, as shown in Figure 8(d). However, residual vibrations of the link are not perfectly suppressed as the simulation results plotted in Figure 4(d). Unwanted residual vibrations occur after joint motion. This is because the joint motor is not capable of absolutely tracking the given reference trajectory as assumed and tracking error is unavoidable. In addition, modeling error also exists in simulation.

The last experiment was the combination of optimal trajectory and feedback control of the PZT actuators. Those experimental results are presented in Figure 9. As the joint trajectory, velocity, and acceleration were not significantly changed, these results were not given. The vibration response of the link is presented in Figure 9(a). It can be seen that the peak value of the link vibrations still exceeds the range of the A/D converter during joint motion, but the residual vibrations, which can be explained by the tracking error and unmodelled high frequency characteristics of the link, are significantly suppressed by the combined effect of the optimal trajectory and active control of PZT actuators. Moreover, the results obtained from the last experiment are compared with those obtained from the above two experiments. Both the peak value and settling time of the measured vibration response after joint motion are given in Table 3.
TABLE 3: Comparison of the experimental results.

\begin{tabular}{lcc}
\hline & $\begin{array}{c}\text { Peak value after } \\
\text { joint motion (V) }\end{array}$ & $\begin{array}{c}\text { Settling time after } \\
\text { joint motion (s) }\end{array}$ \\
\hline $\begin{array}{l}\text { Quintic polynomial } \\
\text { trajectory }\end{array}$ & $>10$ & 9 \\
\hline $\begin{array}{l}\text { Optimal trajectory without } \\
\text { PZT control }\end{array}$ & 1.28 & 3.8 \\
\hline $\begin{array}{l}\text { Optimal trajectory with } \\
\text { PZT control }\end{array}$ & 0.72 & 2.3 \\
\hline
\end{tabular}

As shown in Table 3, it can be observed that residual vibration of the link obtained from the proposed approach is only $0.72 \mathrm{~V}$, less than 10 percent of those obtained by assuming that the trajectory corresponds to the quintic polynomial motion. Meanwhile, compared with the results along the quintic polynomial trajectory, the settling time of the residual vibrations along the optimal trajectory descends approximately 75 percent with the active control of the PZT actuators, reducing to $2.3 \mathrm{~s}$ from $9 \mathrm{~s}$. As a conclusion, the proposed optimal trajectory planning approach is effective for vibration suppression of the flexible manipulator system.

\section{Conclusions}

The above sections have presented an optimal trajectory planning approach for vibration suppression of a flexible piezoelectric manipulator system with bonded PZT actuators. The proposed approach is a combination of the feedforward trajectory planning method and L-type velocity feedback control of piezoelectric actuators based on the Lyapunov approach. The dynamic equations of the link are derived using Hamilton's principle and assumed mode method. In the procedure of trajectory planning, the flexible piezoelectric manipulator system is taken as a whole; rigid motion of the joint, elastic vibration of the link, and active control of the PZT actuators are all considered. Specifically, the joint controller is responsible for trajectory tracking and gross vibration suppression of the link during motion, while the active controller of actuators is expected to deal with residual vibrations after joint motion. The joint angle of the link is expressed as a quintic polynomial function. And the sum of the link vibration energy is chosen as the objective function. Then, the optimal trajectory planning approach is constructed using genetic algorithm. Simulation and experimental results validate the effectiveness of the proposed method. Both the settling time and peak value of the link vibrations along the optimal trajectory descend significantly, with the active control of the PZT actuators. As a conclusion, the proposed optimal trajectory planning approach is effective for vibration suppression of the flexible piezoelectric manipulator system. 


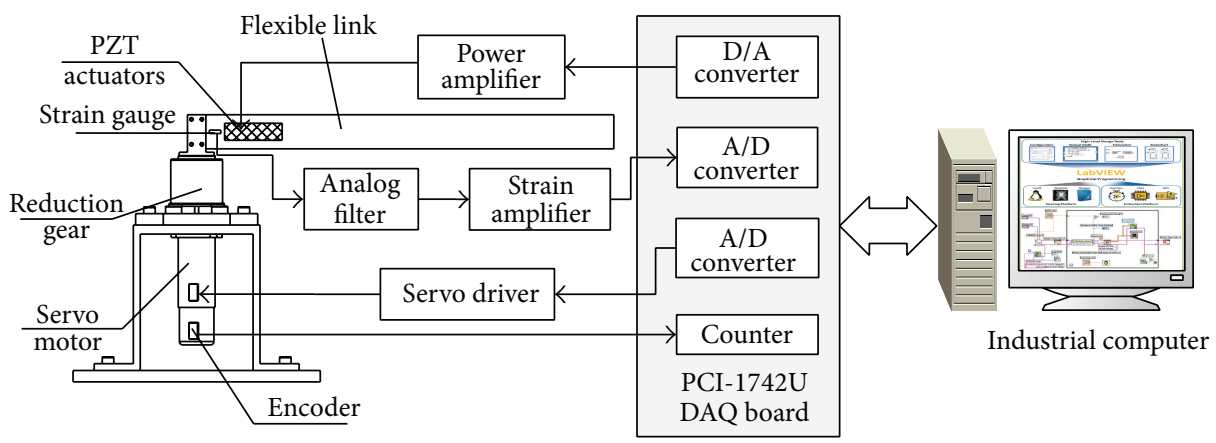

FIGURE 6: Schematic diagram of the experimental setup.

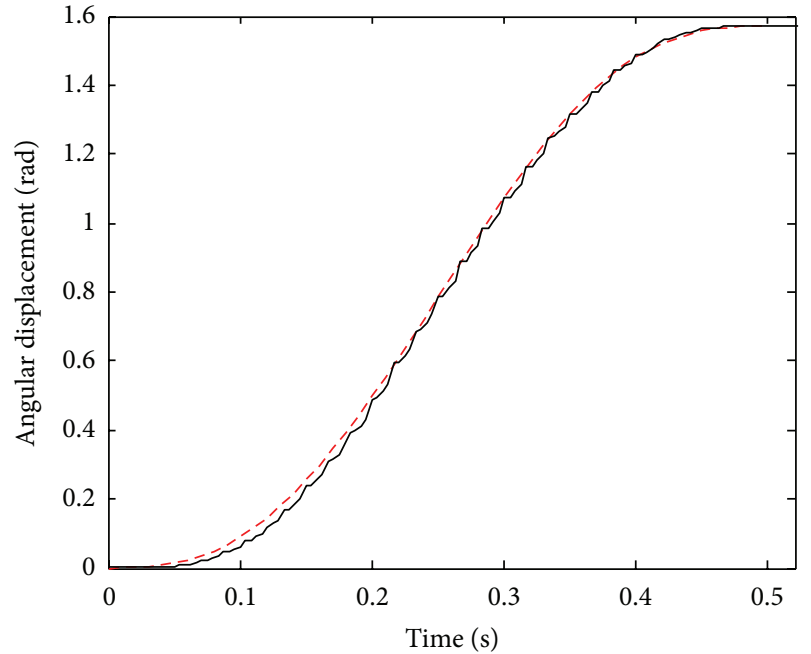

- - - Desired trajectory

- Actual trajectory

(a) Angular displacement

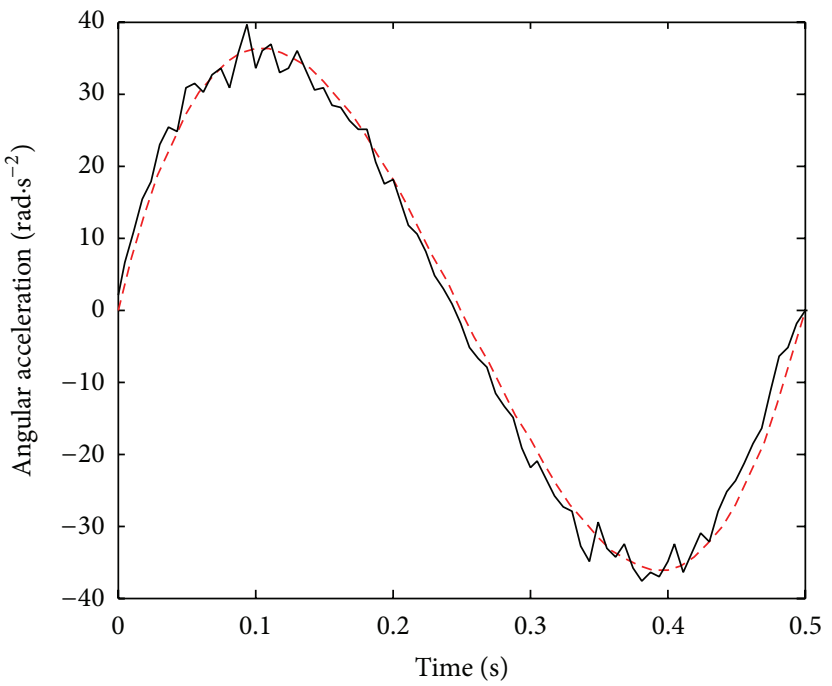

Desired acceleration

Actual acceleration

(c) Angular acceleration

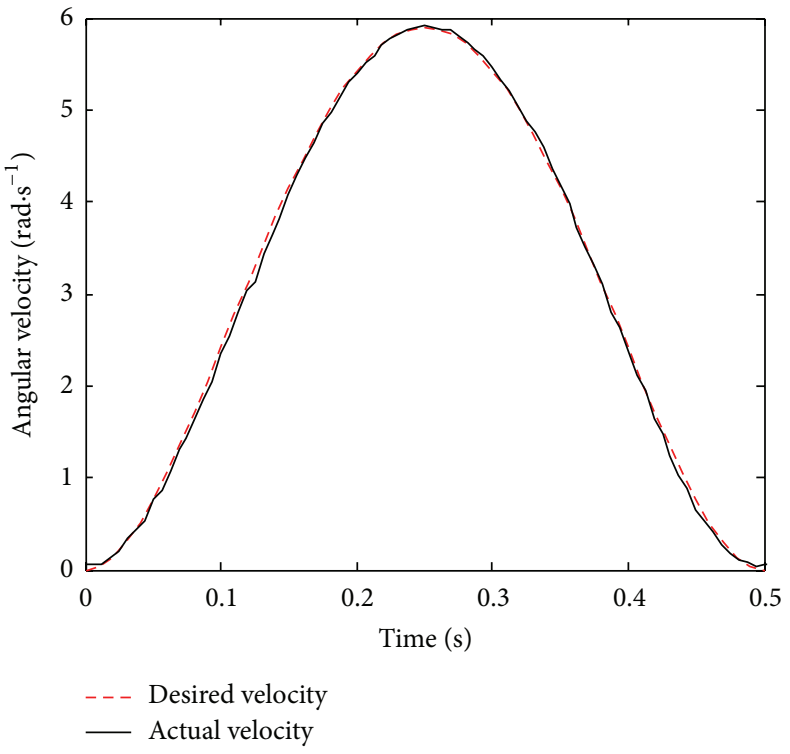

(b) Angular velocity

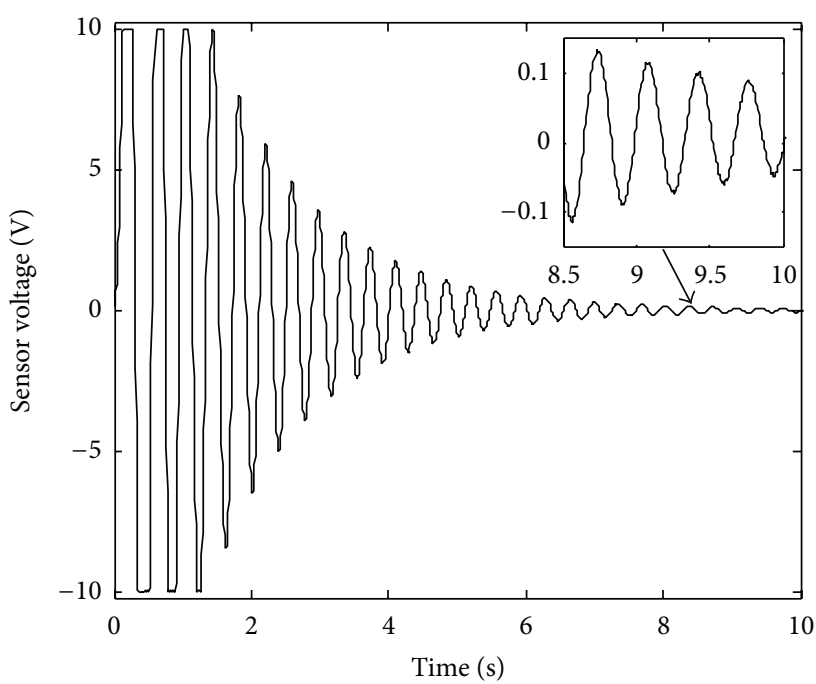

(d) Vibration response of the link

FIgURE 7: Experimental results of the link along the quintic polynomial trajectory. 


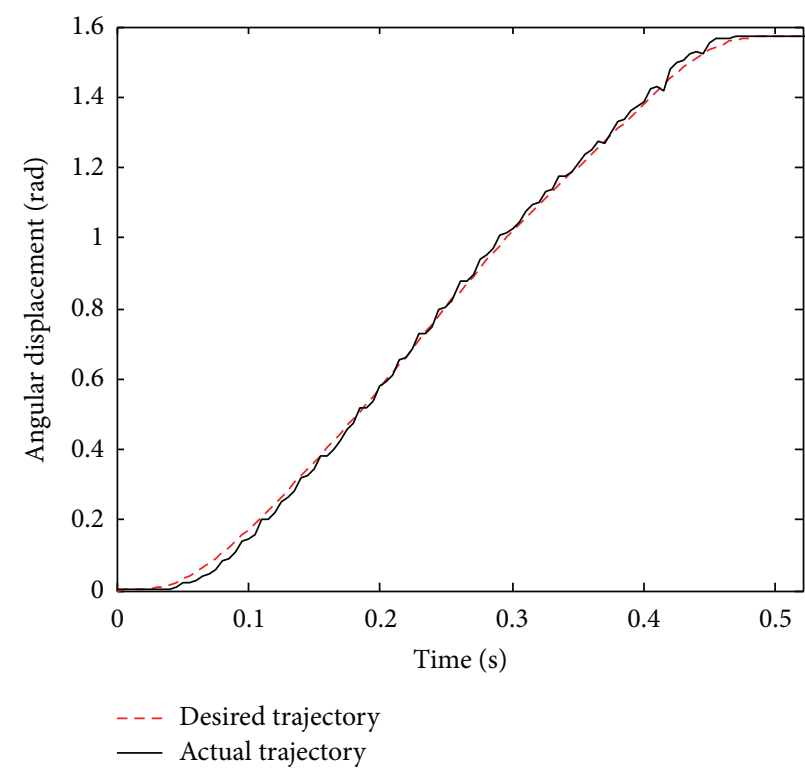

(a) Angular displacement

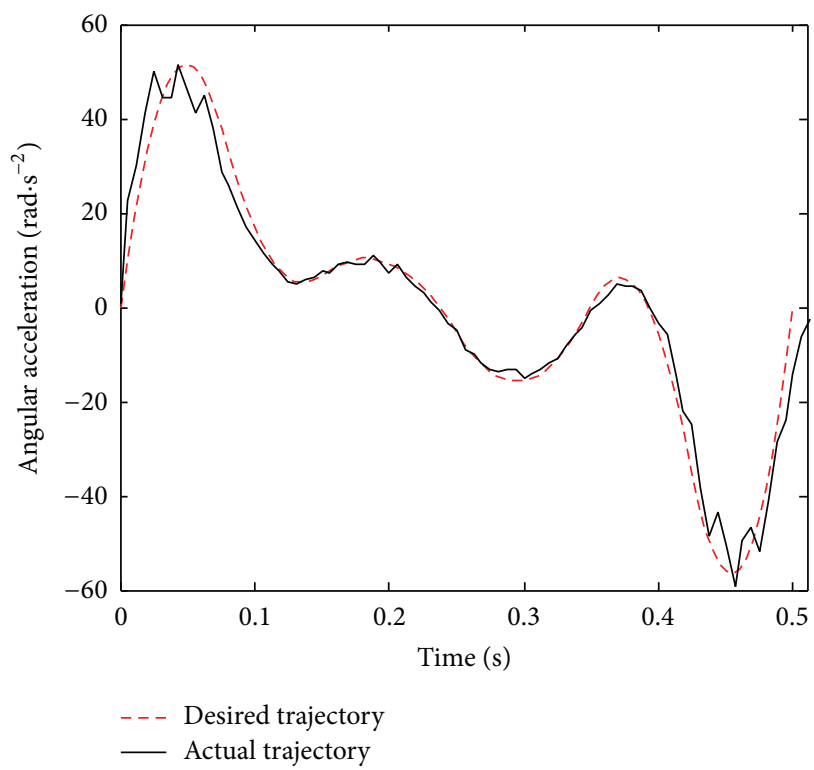

(c) Angular acceleration

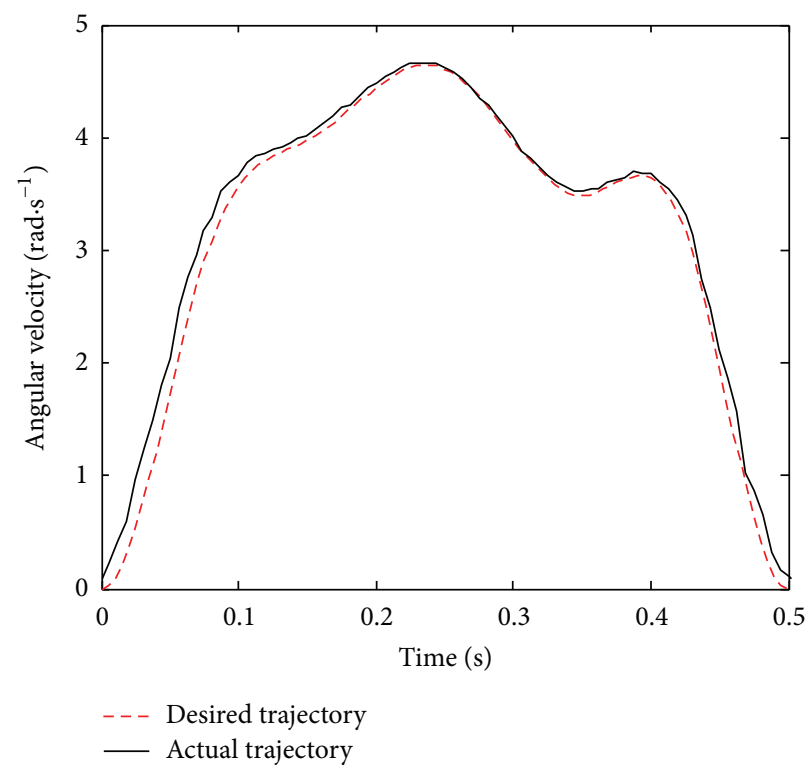

(b) Angular velocity

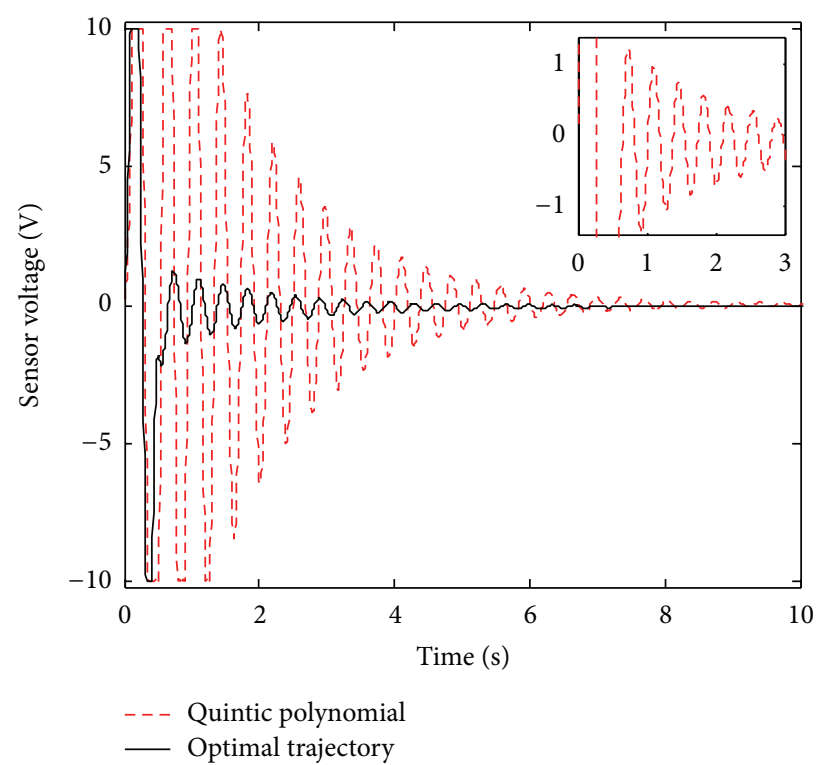

(d) Vibration response of the link

FIgURE 8: Experimental results of the link along the optimal trajectory.

\section{Appendix}

The position vector $\mathbf{p}$ of the link relative to the inertial frame at an arbitrary location $x$ can be expressed as

$$
\mathbf{p}=\left[\begin{array}{l}
p_{x} \\
p_{y}
\end{array}\right]=\left[\begin{array}{l}
(r+x) \cos \theta(t)-w(x, t) \sin \theta(t) \\
(r+x) \sin \theta(t)+w(x, t) \cos \theta(t)
\end{array}\right],
$$

where $r$ is the radius of the rigid hub, $\theta(t)$ is the joint angle, and $w(x, t)$ is the elastic deflection of the link.
The kinetic energy and the potential energy of the flexible manipulator system can be written as

$$
\begin{aligned}
T= & \frac{1}{2} \int_{0}^{l_{b}} \rho_{b} A_{b}(x)\left(\dot{p}_{x}^{2}+\dot{p}_{y}^{2}\right) d x \\
& +\frac{1}{2} \int_{x_{s}}^{x_{s}+l_{p}} 2 \rho_{p} A_{p}\left(\dot{p}_{x}^{2}+\dot{p}_{y}^{2}\right) d x, \\
V_{p}= & \frac{1}{2} \int_{0}^{l_{b}} E_{b} I_{b}\left\{w^{\prime \prime}(x, t)\right\}^{2} d x \\
& +\frac{1}{2} \int_{x_{s}}^{x_{s}+l_{p}} E_{p} I_{p}\left\{w^{\prime \prime}(x, t)\right\}^{2} d x .
\end{aligned}
$$




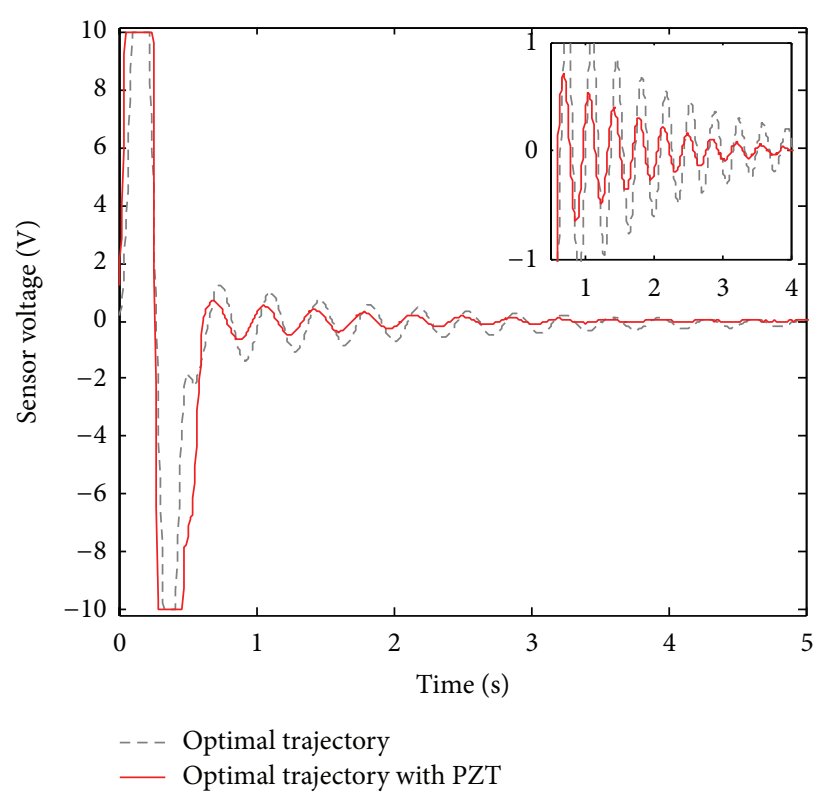

(a) Vibration response of the link

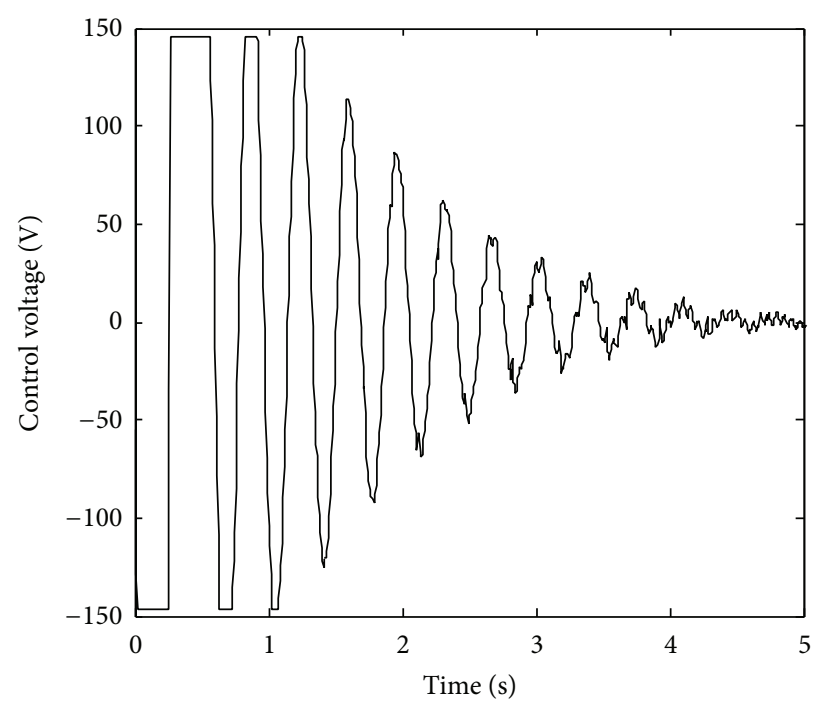

(b) Control voltage of the PZT actuators

FIGURE 9: Experimental results of the link along the optimal trajectory with PZT control.

Here, $\rho A$ is the effective mass per unit length of the structure. $\rho_{b}, A_{b}, l_{b}$ and $\rho_{p}, A_{p}, l_{p}$ devote the density, cross-sectional area, and length of the link and PZT actuators, respectively. $x_{s}$ is the start coordinate of the PZT actuators to the clamped side. And $E I$ is the equivalent flexural rigidity of the link with PZT actuators. $E_{b}, I_{b}$ and $E_{p}, I_{p}$ devote the modulus of elasticity, the moment of inertia of the link, and PZT actuators, respectively.

The virtual work carried out by the moment $M(t)$ of PZT actuators and the input torque acting on the hub $\tau(t)$ can be evaluated as

$$
\delta W=\tau(t) \delta \theta(t)+M(t) \delta w^{\prime}\left(x_{s}, t\right) .
$$

As each PZT actuator is boned to each side of the link, the bending moment $M(t)$ resulting from the voltage applied to the PZT actuators is given by

$$
M(t)=-2 E_{p} d_{31} b_{p} h_{p} V(t)=c V(t),
$$

where $d_{31}$ is the piezoelectric constant, $b_{p}$ and $h_{p}$ are the width and thickness of the PZT actuators respectively, and $V(t)$ is a uniform control voltage applied to the PZT actuators.

Extended Hamilton's principle is used to derive the system equation. Hamilton's principle is given by the following variational statement:

$$
\int_{t_{1}}^{t_{2}}(\delta T-\delta V+\delta W) d t=0
$$

Substituting (A.1) (A.3), incorporating (1) into (A.5), the governing equations of the system can be derived as

$$
\begin{aligned}
& I_{\theta \theta} \ddot{\theta}+\mathbf{q}^{T} \mathbf{m}_{q q} \mathbf{q} \ddot{\theta}+2 \dot{\mathbf{q}}^{T} \mathbf{m}_{q q} \mathbf{q} \dot{\theta}+\mathbf{m}_{\theta q} \ddot{\mathbf{q}}=\tau(t), \\
& \mathbf{m}_{q q} \ddot{q}+\mathbf{m}_{\theta q}^{T} \ddot{\theta}+\mathbf{K}_{q} \mathbf{q}-\mathbf{m}_{q q} \mathbf{q} \dot{\theta}^{2}=c V(t)\left[\widehat{\boldsymbol{\Phi}}^{\prime}\right]^{T}\left(x_{s}\right),
\end{aligned}
$$

where the corresponding coefficients are defined as

$$
\begin{aligned}
I_{\theta \theta}= & \frac{1}{3} \rho_{b} A_{b}\left[\left(r+l_{b}\right)^{3}-r^{3}\right] \\
& +\frac{1}{3} 2 \rho_{p} A_{p}\left[\left(r+x_{s}+l_{p}\right)^{3}-\left(r+x_{s}\right)^{3}\right] \\
\mathbf{m}_{q q}= & \int_{0}^{l_{b}} \rho_{b} A_{b} \boldsymbol{\Phi}^{T}(x) \boldsymbol{\Phi}(x) d x \\
& +\int_{x_{s}}^{x_{s}+l_{p}} \rho_{p} A_{p} \boldsymbol{\Phi}^{T}(x) \boldsymbol{\Phi}(x) d x \\
\mathbf{m}_{\theta q}= & \int_{0}^{l_{b}} \rho_{b} A_{b}(x+r) \boldsymbol{\Phi}(x) d x \\
& +\int_{x_{s}}^{x_{s}+l_{p}} \rho_{p} A_{p}(x+r) \boldsymbol{\Phi}(x) d x \\
\mathbf{K}_{q}= & \int_{0}^{l_{b}} E_{b} I_{b}\left[\boldsymbol{\Phi}^{\prime \prime}\right]^{T}(x) \boldsymbol{\Phi}^{\prime \prime}(x) d x \\
& +\int_{x_{s}}^{x_{s}+l_{p}} E_{p} I_{p}\left[\Phi^{\prime \prime}\right]^{T}(x) \boldsymbol{\Phi}^{\prime \prime}(x) d x \\
\widehat{\Phi}\left(x_{s}\right)= & \boldsymbol{\Phi}\left(x_{s}+l_{p}\right)-\boldsymbol{\Phi}\left(x_{s}\right) .
\end{aligned}
$$

\section{Conflict of Interests}

The authors declare that there is no conflict of interests regarding the publication of this paper. 


\section{Acknowledgments}

This work was supported by the National Natural Science Foundation of China (no. 51375433) and Zhejiang Provincial Natural Science Foundation of China (Grant nos. LY13E050008 and LQ15E050002). The authors would also like to thank the K. C. Wong Magna Fund in Ningbo University for its support.

\section{References}

[1] C. T. Kiang, A. Spowage, and C. K. Yoong, "Review of control and sensor system of flexible manipulator," Journal of Intelligent and Robotic Systems: Theory and Applications, vol. 77, no. 1, pp. 187-213, 2014.

[2] M. Chu, G. Chen, Q.-X. Jia, X. Gao, and H.-X. Sun, "Simultaneous positioning and non-minimum phase vibration suppression of slewing flexible-link manipulator using only joint actuator," Journal of Vibration and Control, vol. 20, no. 10, pp. 1488-1497, 2013.

[3] W. Singhose, "Command shaping for flexible systems: a review of the first 50 years," International Journal of Precision Engineering and Manufacturing, vol. 10, no. 4, pp. 153-168, 2009.

[4] Z. Mohamed, A. K. Chee, A. W. I. Mohd Hashim, M. O. Tokhi, S. H. M. Amin, and R. Mamat, "Techniques for vibration control of a flexible robot manipulator," Robotica, vol. 24, no. 4, pp. 499$511,2006$.

[5] M. S. Alam and M. O. Tokhi, "Designing feedforward command shapers with multi-objective genetic optimisation for vibration control of a single-link flexible manipulator," Engineering Applications of Artificial Intelligence, vol. 21, no. 2, pp. 229-246, 2008.

[6] M. O. T. Cole and T. Wongratanaphisan, "A direct method of adaptive FIR input shaping for motion control with zero residual vibration," IEEE/ASME Transactions on Mechatronics, vol. 18, no. 1, pp. 316-327, 2013.

[7] H. Wu, F. C. Sun, Z. Q. Sun, and L. C. Wu, "Optimal trajectory planning of a flexible dual-arm space robot with vibration reduction," Journal of Intelligent and Robotic Systems, vol. 40, no. 2, pp. 147-163, 2004.

[8] H. R. Heidari, M. H. Korayem, M. Haghpanahi, and V. F. Batlle, "Optimal trajectory planning for flexible link manipulators with large deflection using a new displacements approach," Journal of Intelligent and Robotic Systems: Theory and Applications, vol. 72, no. 3-4, pp. 287-300, 2013.

[9] K. Springer, H. Gattringer, and P. Staufer, "On time-optimal trajectory planning for a flexible link robot," Proceedings of the Institution of Mechanical Engineers, Part I: Journal of Systems and Control Engineering, vol. 227, no. 10, pp. 752-763, 2013.

[10] Y. Choi, J. Cheong, and H. Moon, "A trajectory planning method for output tracking of linear flexible systems using exact equilibrium manifolds," IEEE/ASME Transactions on Mechatronics, vol. 15, no. 5, pp. 819-826, 2010.

[11] J. Lessard, P. Bigras, Z. Liu, and B. Hazel, "Characterization, modeling and vibration control of a flexible joint for a robotic system," Journal of Vibration and Control, vol. 20, no. 6, pp. $943-$ 960, 2014.

[12] M. Moallem, M. R. Kermani, R. V. Patel, and M. Ostojic, "Flexure control of a positioning system using piezoelectric transducers," IEEE Transactions on Control Systems Technology, vol. 12, no. 5, pp. 757-762, 2004.

[13] V. B. Nguyen and A. S. Morris, "Genetic algorithm tuned fuzzy logic controller for a robot arm with two-link flexibility and two-joint elasticity," Journal of Intelligent and Robotic Systems: Theory and Applications, vol. 49, no. 1, pp. 3-18, 2007.

[14] J. Das and N. Sarkar, "Passivity-based target manipulation inside a deformable object by a robotic system with noncollocated feedback," Advanced Robotics, vol. 27, no. 11, pp. 861$875,2013$.

[15] C.-Y. Lin and C.-M. Chang, "Hybrid proportional derivative/repetitive control for active vibration control of smart piezoelectric structures," Journal of Vibration and Control, vol. 19, no. 7, pp. 992-1003, 2013.

[16] J.-J. Wei, Z.-C. Qiu, J.-D. Han, and Y.-C. Wang, "Experimental comparison research on active vibration control for flexible piezoelectric manipulator using fuzzy controller," Journal of Intelligent and Robotic Systems: Theory and Applications, vol. 59, no. 1, pp. 31-56, 2010.

[17] D. Sun, J. K. Mills, J. J. Shan, and S. K. Tso, "A PZT actuator control of a single-link flexible manipulator based on linear velocity feedback and actuator placement," Mechatronics, vol. 14, no. 4, pp. 381-401, 2004.

[18] K. Gurses, B. J. Buckham, and E. J. Park, "Vibration control of a single-link flexible manipulator using an array of fiber optic curvature sensors and PZT actuators," Mechatronics, vol. 19, no. 2, pp. 167-177, 2009.

[19] L. Y. Li, G. B. Song, and J. P. Ou, "Adaptive fuzzy sliding mode based active vibration control of a smart beam with mass uncertainty," Structural Control and Health Monitoring, vol. 18, no. 1, pp. 40-52, 2011.

[20] S.-B. Choi, M.-S. Seong, and S. H. Ha, "Accurate position control of a flexible arm using a piezoactuator associated with a hysteresis compensator," Smart Materials and Structures, vol. 22, no. 4, Article ID 045009, 2013.

[21] Y. S. Bian and Z. H. Gao, "Impact vibration attenuation for a flexible robotic manipulator through transfer and dissipation of energy," Shock and Vibration, vol. 20, no. 4, pp. 665-680, 2013.

[22] A. A. Ata, "Inverse dynamic analysis and trajectory planning for flexible manipulator," Inverse Problems in Science and Engineering, vol. 18, no. 4, pp. 549-566, 2010.

[23] M. D. G. Marcos, J. A. Tenreiro Machado, and T.-P. AzevedoPerdicoúlis, "A multi-objective approach for the motion planning of redundant manipulators," Applied Soft Computing Journal, vol. 12, no. 2, pp. 589-599, 2012.

[24] T. Nestorović and M. Trajkov, "Optimal actuator and sensor placement based on balanced reduced models," Mechanical Systems and Signal Processing, vol. 36, no. 2, pp. 271-289, 2013.

[25] W. C. Sun, H. J. Gao, and O. Kaynak, "Adaptive backstepping control for active suspension systems with hard constraints," IEEE/ASME Transactions on Mechatronics, vol. 18, no. 3, pp. 1072-1079, 2013. 

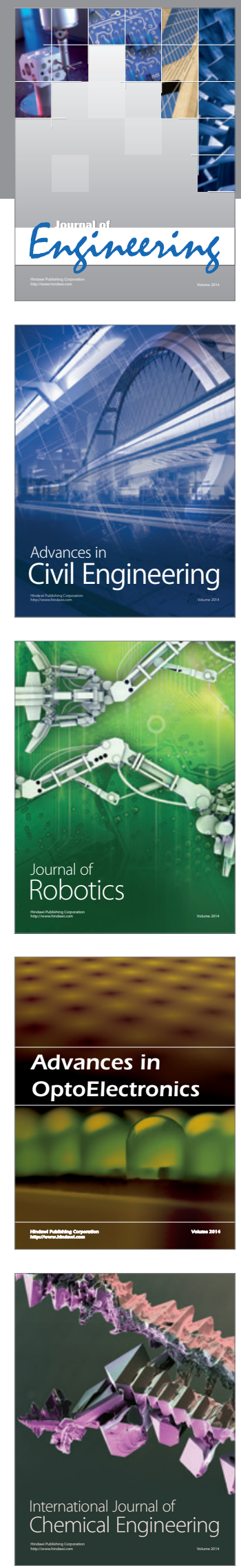

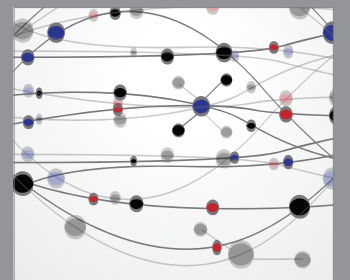

The Scientific World Journal
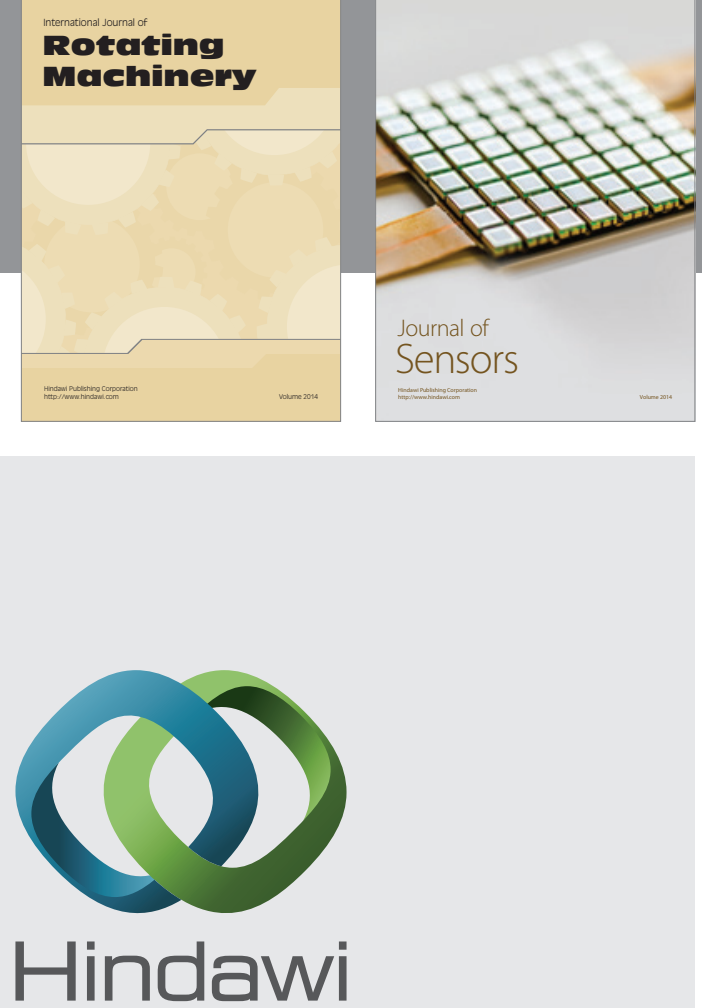

Submit your manuscripts at http://www.hindawi.com
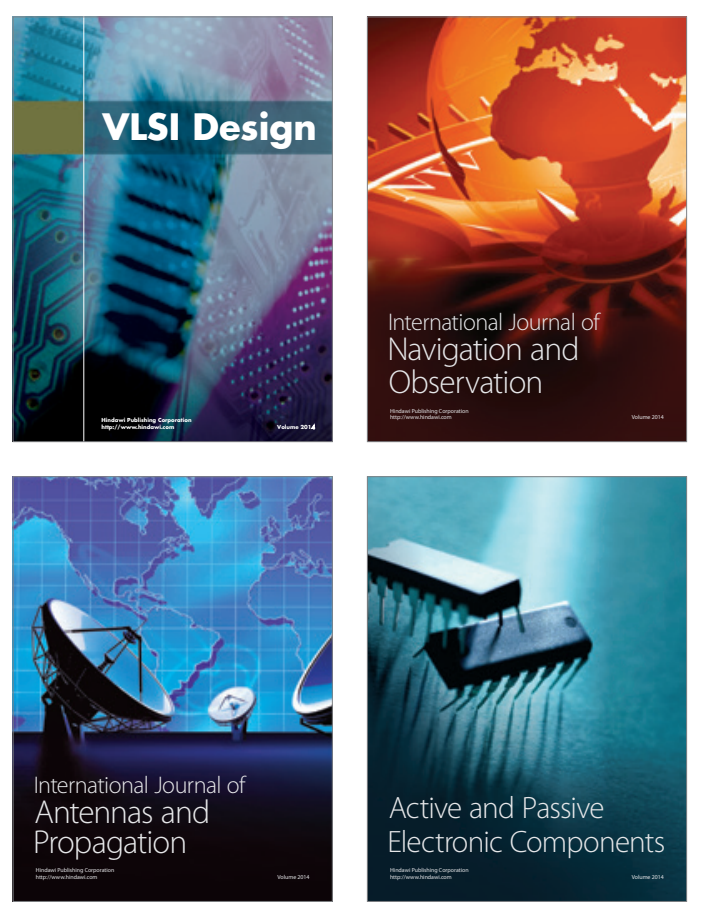
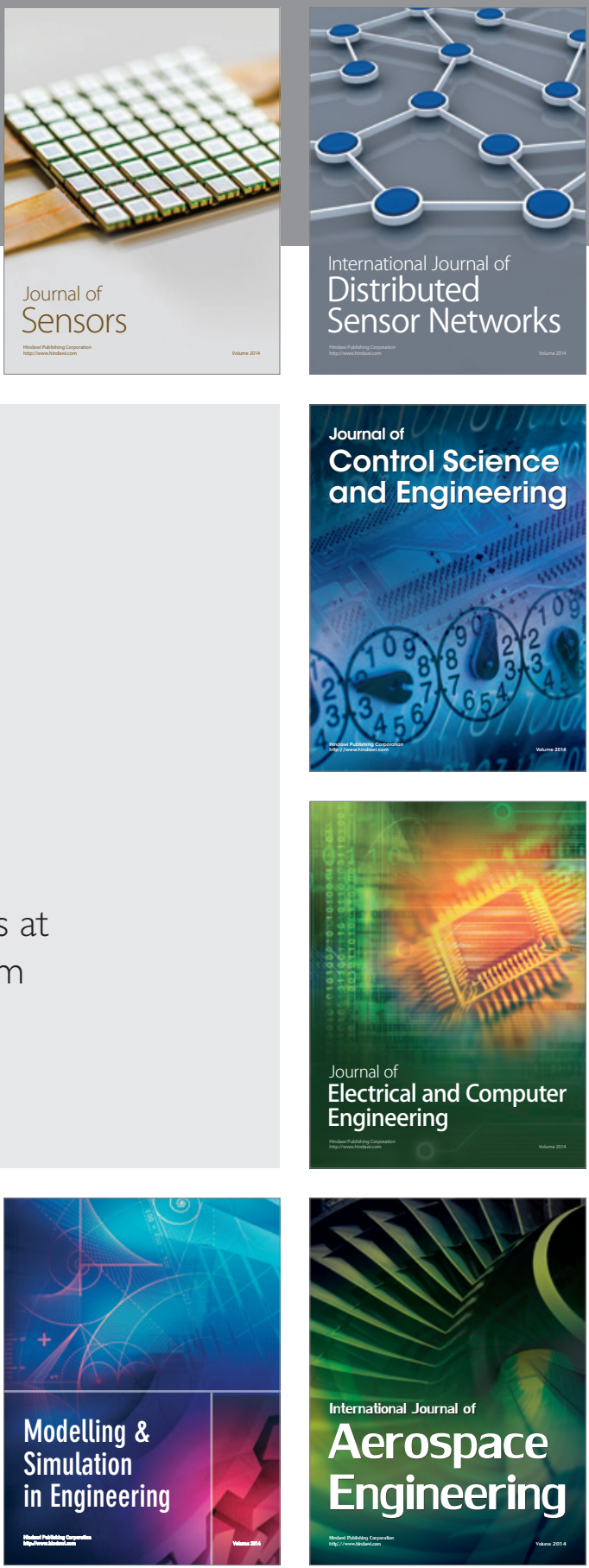

Journal of

Control Science

and Engineering
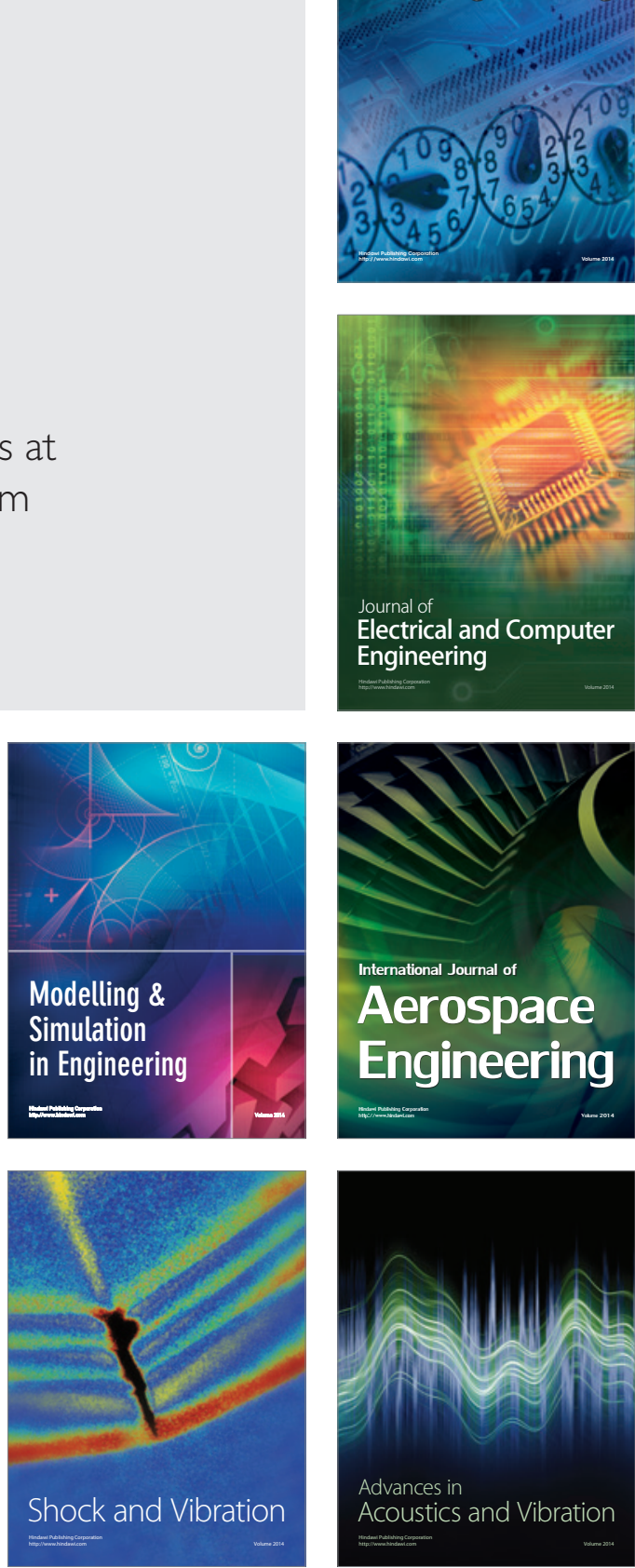